\title{
FUZZY LOGIC APPROACH IN MODE CHOICE MODELLING FOR EDUCATION TRIPS: A CASE STUDY OF INDIAN METROPOLITAN CITY
}

\author{
Ashu Shivkumar Kedia ${ }^{1}$, Krishna Bhuneshwar Saw ${ }^{2}$, Bhimaji Krishnaji Katti ${ }^{3}$ \\ ${ }^{1}$ Pandit Deendayal Petroleum University, Raisan, Gandhinagar, Gujarat, India \\ ${ }^{2}$ Institute of Urban Transport, New Delhi, India \\ ${ }^{3}$ Sardar Vallabh Bhai National Institute of Technology, Surat, Gujarat, India
}

Submitted 26 September 2014; resubmitted 13 November 2014; accepted 15 January 2015

\begin{abstract}
Urban population in India has increased significantly from 62 million in 1951 to 378 million in 2011 in six decades. It is estimated to reach 540 million by the year 2021. This reflects on likely pressure on urban transportation system. The situation necessarily calls plans for balanced personal and public transport system. Mandatory trips bear more importance in this regard owing to their higher share in urban trips. Mode share and their choice behaviour in estimation of such trips play vital role in analysing and boosting sustainable transportation. Logit modelling approach is the conventional method generally adopted for analysing mode choice behaviour, which is based on the principle of random utility maximization derived from econometric theory. However, such models cannot address uncertainity prevailing in the choice decisions. On the contrary, fuzzy logic bypasses the binary crisp derivations of the inputs and accepts multivalued inputs in linguistic expressions, which make possible to resemble the human behaviour closely. Therefore, the attempt here is to develop fuzzy logic based mode choice model for education trips, which constitutes a good share in mandatory trips by covering various income groups of Indian society.
\end{abstract}

Keywords: fuzzy logic; mode choice; rule-based system; education trips; urban travel.

\section{Introduction}

Industrialization in the recent years in developing economies like India has caused a large mass of people from rural area to migrate and get settled in the vicinity of the trade and commerce centres, thereby forming urban agglomerations. Consequently, the number of metropolitan cities in India with a population exceeding one million has increased from 35 in 2001 to 44 in 2011. It is interesting to note here that nearly $65 \%$ of the urban population is concentrated in class-I cities and $32 \%$ of this further is in metropolitan centers itself. Owing to good job potential along with the increased mobility requirements have led to the intensive motorization in the urban areas. Nearly 250 to 600 motor vehicles are getting added daily in metropolitan centers in the country. Interestingly, these mainly comprise of two-wheelers, three-wheelers and cars with significant growth in two-wheelers. This has resulted into increase in traffic congestion, environmental pollution, road accidents and parking problems. The situation calls for probing and understanding the mode choice phenomenon on part of the urbanites, so as to formulate and initiate appropriate transport policies.
In fact, mode choice analysis is the third phase of sequential urban travel demand forecasting process after trip generation and distribution stages. It is generally explained by three basic factors: characteristics of the journey, socio-economic characteristics of the traveller, and the transport system. Moreover, mode choice predictions bear importance in framing transportation system policies and congestion mitigation strategies apart from the impact on urban transport economics, road space sharing, traffic congestion, road safety. In simple terms, it does have an effect on traffic and travel quality. The traditional models cannot deal effectively with ambiguities, uncertainties, and vagueness prevailing in human decisions or perception of utility values. Therefore, the use of soft computing techniques bears importance due to their ability to handle quantitative and qualitative measures under uncertainty. These have some similarities to cognitive mechanisms applied by individuals in the process of choice making and problem solving. Fuzzy logic, as a soft computing tool can be employed to address effectively the uncertainty prevailing in the decision process. Therefore, the research focus here has been to tackle the urban transport mode choice behaviour for 
commuting educational trips, which are significant next to work trips in a metropolitan environment through fuzzy system.

\section{Literature Base}

In the recent past, researchers have sought to apply fuzzy logic for mode choice modelling. Fuzzy logic theory was initiated by Zadeh (1965). Mamdani and Assilian (1975) adopted fuzzy logic in linguistic synthesis. In late 1980s a group of Japanese authors made significant contributions to fuzzy set theory applications in machine control system and traffic and transportation. The process to develop fuzzy rules from numerical data was put forward by Wang and Mendel (1992), which was further utilized by Kalić and Teodorović $(1996,2003)$ to solve various transportation problems and specially the mode choice analysis using fuzzy reasoning. Utility by travel mode was represented through fuzzy system rather than considering it as a deterministic concept by Teodorović (1999). Holland (2000) determined the modal split using a fuzzy inference system, which was adopted to determine the preference for a given set of perceived journey characteristics. A combined fuzzy-logit model using knowledge-based approach came into picture from the study done by Mizutani and Akiyama (2000). A study later by the same authors concluded that logit-models with fuzzy reasoning-based utility functions can describe a human decision with vagueness explicitly. Vythoulkas and Koutsopoulos (2003) advocated the use of fuzzy logic in capturing steady state and long-term behavior as well. The intuitive and interpretable nature of the rule base makes Mamdani fuzzy inference system widely useful as per Mendel (2001) and Jassbi et al. (2007). Fuzzy based models have upper edge over the traditional logitmodels to address the uncertainty lying in the choice making behaviour of travellers as per the observations of Errampalli et al. (2011) in his research work. Murat and Uludag (2008) concluded fuzzy logic based route choice model to behave more realistically because of its characteristics on modelling imprecision and uncertainty lying in the human perceptions. Bataineh et al. (2011) compared the two most famous clustering techniques: fuzzy c-means algorithm and subtractive clustering algorithm based on validity measurement of their clustering results for highly non-linear functions. Another application of fuzzy logic - subtractive clustering technique was demonstrated by Pulugurta et al. (2014) in developing travel demand models at different stages of four-stage modelling. Fuzzy logic based mode choice models are also advocated for better prediction over the traditional multi nominal logit-model by Dell'Orco, Ottomanelli (2012) and Pulugurta et al. (2013). Though the fuzzy logic based model development is a time consuming process, it was observed offering a significant flexibility in evaluating any kind of sensitive policy pertaining to transit demand by Kumar et al. (2013). Kompil and Celik (2013) came up with the genetic-fuzzy hybrid approach for trip distribution modelling where simple fuzzy rule-based system and a novel genetic fuzzy rule-based system (a fuzzy system improved by a knowledge base learning process with genetic algorithms) were introduced. Although it brings an additional computation cost, use of genetic algorithms in fuzzy rule-based learning was observed to increase the modelling performance considerably. The hybrid approach using fuzzy logic with neural network or genetic algorithm is now gaining due importance to improve over the results in recent times.

In Indian metropolitan context, a huge diversity prevails in socio-economic profiles and consequently in the choice sets. Therefore, fuzzy logic plays significant role in modelling the travel behaviour in our metropolitan context.

\section{Study Area and Database}

Athwa zone, fast growing residential area of Surat, an industrial city in South Gujarat region has been considered as the study area (Fig. 1). The city with 4.6 million population covering $326 \mathrm{~km}^{2}$ area is known for its jari, diamond, textile, chemical and fertilizer industries. The decadal growth rate of the city is above $60 \%$ since three decades.

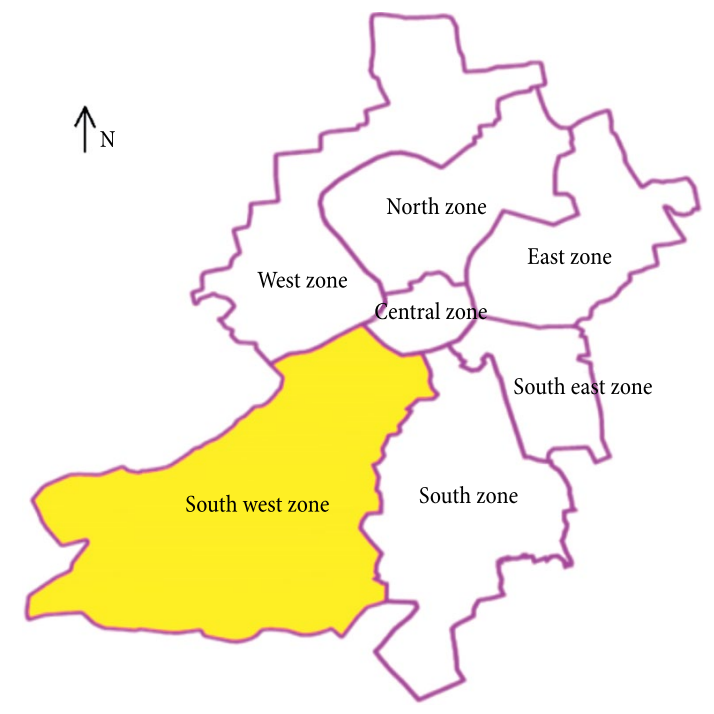

Fig. 1. Study area - Athwa zone (South West) of Surat city

Athwa zone consists of 22 census wards to cover 87000 households in nearly $98 \mathrm{~km}^{2}$ area. The wards are further clubbed to form eight sub-zones for the study purpose. Database has been developed through HomeInterview Surveys carried with pre-designed questionnaire to capture travellers' mode choice decisions. Nearly 650 filtered samples of 900 random household samples covering all the sections of society are considered for modelling purpose. Here, modal choice pertains to five categories: walking, auto-rickshaw, school bus, twowheeler and cars/school vans in absence of desired city bus services at present. The survey data of three major attributes on income levels, trip length and travel comfort and convenience provides the platform for developing the model. Travel cost of the trip is considered indirectly through the segregation of income groups for their choice behaviour. 


\section{Mode Share for Education Trips}

The proportion of education trips among the routine trips is found nearly $40 \%$ for all the three major income classes of 'Low', 'Medium' and 'High' Income Groups (LIG, MIG and HIG, respectively). The mode share observations for them are mentioned in Table 1, wherein the share of auto-rickshaw and two-wheeler is on higher side. Share of non-motorized modes like walking and cycling is negligible for middle and higher income classes. Inadequate facilities for pedestrians and cyclists and insecure feeling may be the possible reason behind their lower share. Shared auto-rickshaws are preferred by 'Low' and 'Low Middle' income groups. Walking is of higher order for LIG category. Sharing of school bus is observed more for higher MIG and HIG groups.

Table 1. Modal share observations

\begin{tabular}{lcccccc}
\hline & $\begin{array}{c}\text { Two- } \\
\text { Wheeler }\end{array}$ & $\begin{array}{c}\text { Auto } \\
\text { Rickshaw }\end{array}$ & Bus & Car & Walk & Total \\
\hline LIG & 17.3 & 41.7 & 4.3 & 2.9 & 33.8 & 100.0 \\
\hline MIG & 37.4 & 29.0 & 12.4 & 3.9 & 17.3 & 100.0 \\
\hline HIG & 44.5 & 19.3 & 19.3 & 13.4 & 3.5 & 100.0 \\
\hline
\end{tabular}

\section{Development of Fuzzy Based Model}

A large fragment of vehicular traffic is usually from education trips after the work trips. Up to secondary level of education, students are generally captive to travel modes and choice decisions are by elders. However, from secondary level onwards they have several alternatives available for the travel mode to commute their education trips. The development and testing of fuzzy inference system based models to capture the choice behaviour were carried out using the fuzzy logic toolbox in the MatLab (version 7.6.0 R2008a).

\subsection{Model Inputs and Outputs}

Fuzzy Rule Based Mode Choice Model for Education trips (FRB-MCM-E) is being developed here with the input variables Household Incomes (HHIs), Trip Lengths (TLs) and Comfort \& Convenience (C\&C) levels. Trip lengths, child security, trip comfort and flexibility in terms of service at the door steps etc. are observed to bear importance in mode choice as revealed in the home interviews and so form the base for inputs in the present study. A small proportion of education trips are commuted by non-motorized modes such as walking and cycling. For short trips, non-motorized modes are observed to be preferred. Thus, trip length instead of travel time is being preferred here in modelling. $\mathrm{C} \& \mathrm{C}$ reflect on door-to-door service and protection from the sun and rain etc. and they are rated in percentage as shown in Table 2. Higher index is for better mode. The ratings are based on degree of convenience, travel time, at door service, inside comfort and safety, etc.
Five options in outputs are walking, auto-rickshaw, bus, two-wheeler, and car/school van. Owing to very less share of cycles, it is not considered as the possible alternative in the present work. The availability of school buses and auto-rickshaws for long trips and preference to walking for short trips of pre-primary and primary education level may be the reason behind small share of bicycle trips.

Table 2. Assumed C\&C index

\begin{tabular}{cc}
\hline Travel mode & C\&C index [\%] \\
\hline Walking & $20-30$ \\
\hline Auto-rickshaw & $30-40$ \\
\hline Bus & $40-50$ \\
\hline Two-wheeler & $50-60$ \\
\hline Car/School van & $60-70$ \\
\hline
\end{tabular}

\subsection{Fuzzy Model Structure}

The fuzzy model operates in three steps as mentioned below and the respective fuzzy logic structure is depicted in Fig. 2.

- fuzzification of crisp input variables through Membership Functions (MFs);

- rule-based inference;

- defuzzification.

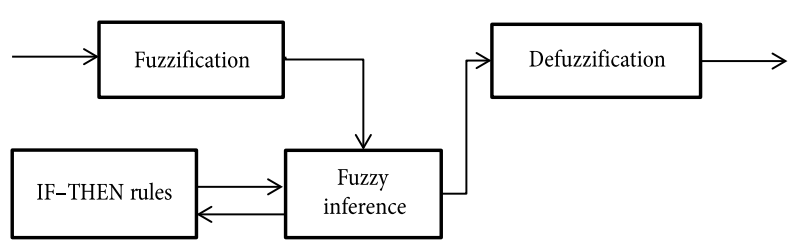

Fig. 2. Fuzzy logic structure

\subsection{Setting of Membership Functions}

The data collected during home interview surveys pertaining to household income, trip lenght, $C \& C$ etc. are rather in crisp forms which need further fuzzification through appropriate membership functions. The triangular or Gaussian functions around a crisp value better reflects the flexible boundary conditions for the inputs. As the former function is simple and does not affect the outcome much as reported in literature is adopted here. The inputs are further categorized into reasonable number of fuzzy sets to reflect on their likely impacts. With this background, the model inputs - HHI, TL and C\&C - are labelled from 'Very Low' to 'Very High' as shown in Fig. 3a to Fig. 3c. The model output which is in form of Travel Modes (TMs) five in number forms the triangular MF (Fig. 3d).

Later, either the left and/or right spreads as well as overlapping of the triangular MFs are set to meet the trend in the empirical data. The final ranges of the input and output fuzzy sets are as shown in Table 3. 

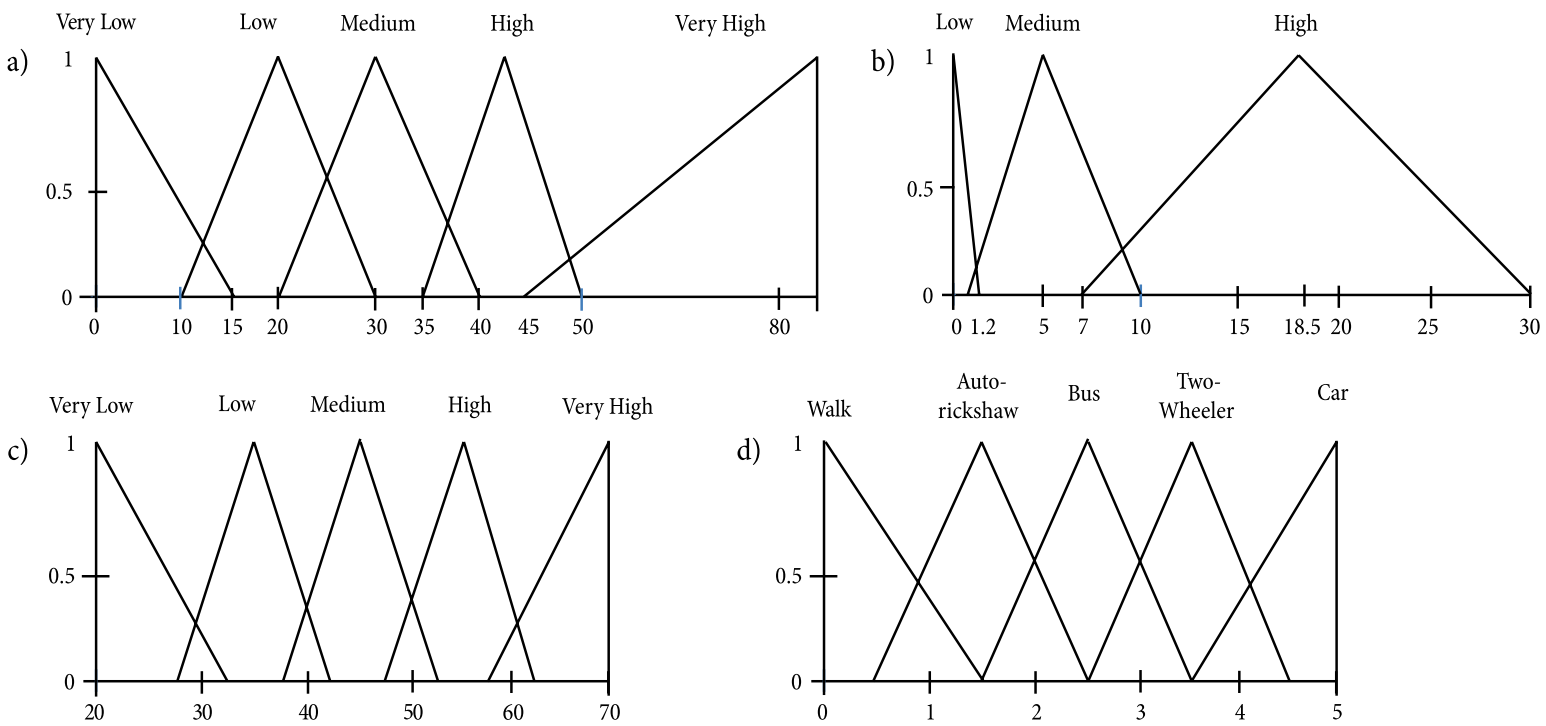

Fig. 3. MFs of input variables: a - Household Income (HHI); b - Trip Length (TL); c - Comfort \& Convenience (C\&C); d - Travel Mode (TM)

Table 3. MF of input variables

\begin{tabular}{|c|c|}
\hline Levels & Range \\
\hline \multicolumn{2}{|c|}{ Household Income $(\mathrm{HHI})^{\star}$} \\
\hline Very Low & {$\left[\begin{array}{lll}0 & 0 & 15\end{array}\right]$} \\
\hline Low & {$\left[\begin{array}{lll}10 & 20 & 30\end{array}\right]$} \\
\hline Medium & {$\left[\begin{array}{lll}20 & 30 & 40\end{array}\right]$} \\
\hline High & {$\left[\begin{array}{lll}35 & 42.5 & 50\end{array}\right]$} \\
\hline Very High & {$\left[\begin{array}{lll}45 & 85 & 85\end{array}\right]$} \\
\hline \multicolumn{2}{|c|}{ Trip Length (TL) $[\mathrm{km}]$} \\
\hline Low & {$\left[\begin{array}{lll}0 & 0 & 1.2\end{array}\right]$} \\
\hline Medium & {$\left[\begin{array}{lll}0.8 & 5 & 10\end{array}\right]$} \\
\hline High & $\left.\begin{array}{llll}7 & 18.5 & 30\end{array}\right]$ \\
\hline \multicolumn{2}{|c|}{ Comfort \& Convenience (C\&C) [\%] } \\
\hline Very Low & {$\left[\begin{array}{lll}20 & 20 & 32.5\end{array}\right]$} \\
\hline Low & {$\left[\begin{array}{llll}27.5 & 35 & 42.5\end{array}\right]$} \\
\hline Medium & {$\left[\begin{array}{lll}37.5 & 45 & 52.5\end{array}\right]$} \\
\hline High & {$\left[\begin{array}{lll}47.5 & 55 & 62.5]\end{array}\right.$} \\
\hline Very High & {$\left[\begin{array}{llll}57.5 & 70 & 70\end{array}\right]$} \\
\hline \multicolumn{2}{|c|}{ Travel Mode (TM) } \\
\hline Walking & {$\left[\begin{array}{lll}0 & 0 & 1.5\end{array}\right]$} \\
\hline Auto-rickshaw & {$\left[\begin{array}{llll}0.5 & 1.5 & 2.5\end{array}\right]$} \\
\hline School bus & {$\left[\begin{array}{llll}1.5 & 2.5 & 3.5\end{array}\right]$} \\
\hline Two-wheeler & {$\left[\begin{array}{llll}2.5 & 3.5 & 4.5\end{array}\right]$} \\
\hline Car & {$\left[\begin{array}{lll}3.5 & 5 & 5\end{array}\right]$} \\
\hline
\end{tabular}

Note: ${ }^{\star}$ income is in thousands INR.

\subsection{Fuzzy Rule Formulation}

Fuzzy rule base for FRB-MCM-E is developed for five income segments, each having 15 numbers of rules with three levels of trip lengths and five levels of comfort \& convenience for one income category.
Fig. 4 shows the typical schematic diagram of fuzzy rule base development for fuzzy set 'Very Low' income. Similarly the rules have been formed for other fuzzy income sets such as 'Low', 'Medium,' 'High' and 'Very High' with different combinations among input variables keeping in view of field observations on modal choice. The rule base is constituted by 75 numbers of IF-THEN rules for five income categories. A typical rule is IF Household income is 'Low' AND Trip length is 'Low' AND Comfort \& convenience is 'Very Low', THEN Mode chosen is 'Walk' Numbers of such rules are framed on appropriate reasoning of mode choice.

\subsection{Defuzzification Process}

As the output generated by fuzzy inference system cannot be used directly, it is necessary to defuzzify the fuzzy quantities into crisp terms for the application purpose. Many defuzzification techniques have been proposed by researchers. The most commonly used method is Centroid method in Mamdani inference mechanism. The method calculates the weighted average of the elements in the support set and analyses the combined shape of membership function, which gives us comparatively good results.

The output membership areas are determined by the height of the membership function on the Y-axis as dictated by the rule strength value obtained by MaxMin function approach. The centroids of the areas marked are computed for each of the competing output membership functions. Subsequently, centre of gravity is computed using the weighted average values which is depicted in MatLab rule viewer window.

The typical output value of 2.5 is for the three specified inputs of income (42500 INR), trip length $(15 \mathrm{~km})$ and comfort \& convenience level (45\%) as shown in Fig. 5 for the possible choice of school bus. Surface plots for predicted travel mode by the developed model are shown below in Fig. 6 . 


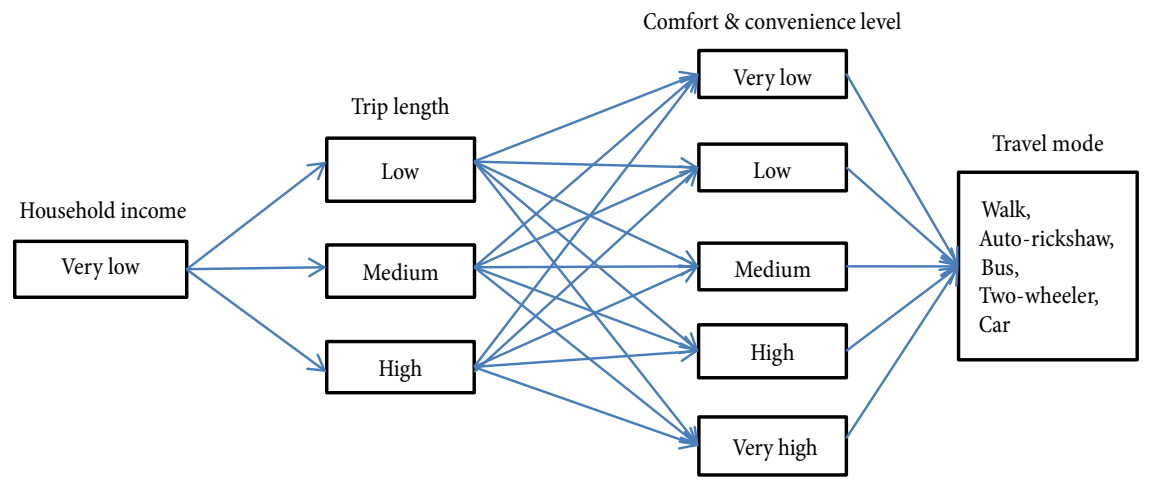

Fig. 4. Typical fuzzy rule base for fuzzy set 'Very Low' income group

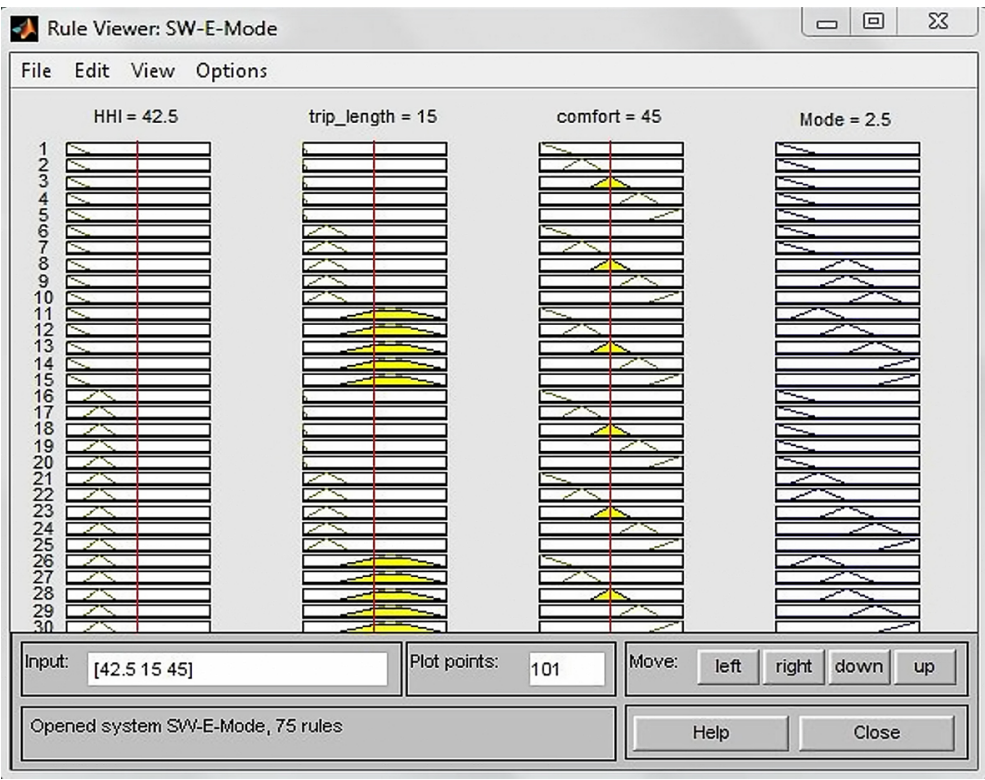

Fig. 5. Typical MatLab snapshot of rule viewer window
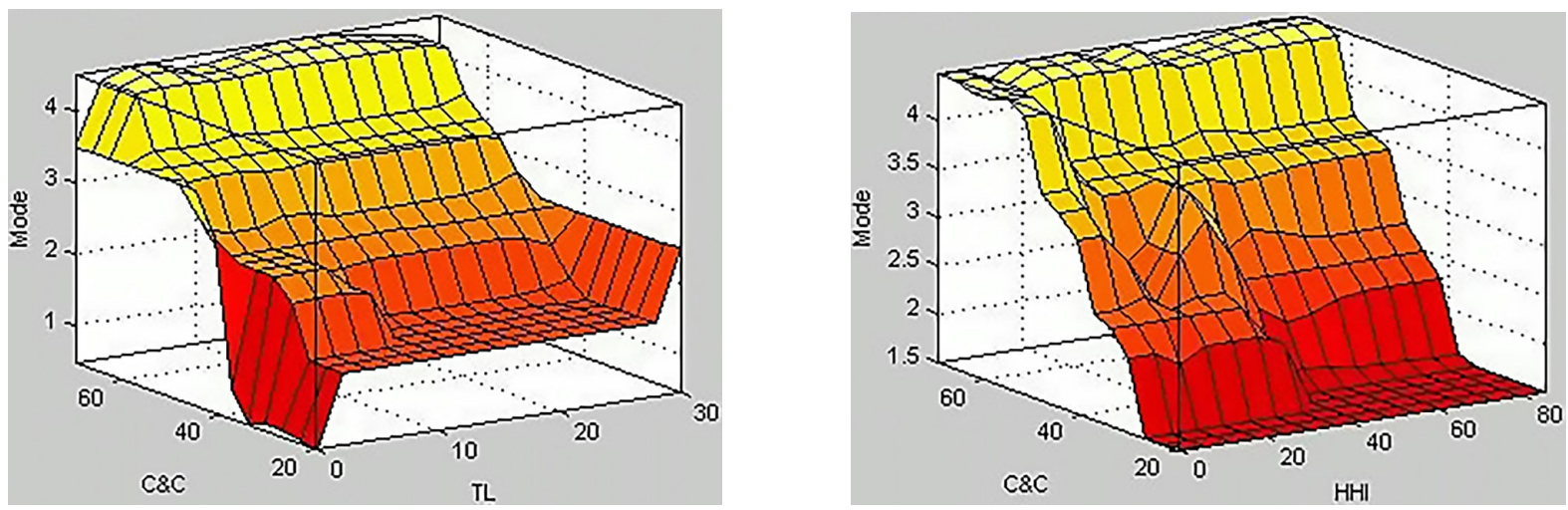

Fig. 6. Surface plots for predicted travel mode

\section{Analysis and Discussion}

\subsection{Calibration and Validation Results}

Field observed and model predicted results are presented in Table 4 for calibration and validation datasets. Accuracy level of $83 \%$ and $91 \%$ while calibrating and validating the dataset respectively has been achieved through the developed model. It can be inferred that the developed model is able to map the mode choice making behaviour on the basis of logical IF-THEN rules.

The comparison of observed modal split and the one predicted by the developed fuzzy rule-based model is shown in Fig. 7. The developed model is capable of predicting the mode used for education purpose in an 
Table 4. Field observed and model predicted mode choice

\begin{tabular}{|c|c|c|c|c|c|c|c|}
\hline \multicolumn{8}{|c|}{ Calibration } \\
\hline \multirow{8}{*}{$\begin{array}{c}\text { Observed } \\
\text { (No. of trips) }\end{array}$} & \multicolumn{7}{|c|}{ Predicted (No. of trips) } \\
\hline & & Walk & Auto-rickshaw & Bus & Two-wheeler & Car & Total \\
\hline & Walk & 25 & 5 & 0 & 0 & 0 & 30 \\
\hline & Auto-rickshaw & 8 & 45 & 6 & 0 & 0 & 59 \\
\hline & Bus & 0 & 1 & 40 & 4 & 0 & 45 \\
\hline & Two-wheeler & 2 & 11 & 3 & 111 & 1 & 128 \\
\hline & Car/School van & 0 & 0 & 0 & 6 & 18 & 24 \\
\hline & Total & 35 & 62 & 49 & 121 & 19 & 286 \\
\hline \multicolumn{8}{|c|}{ Validation } \\
\hline \multicolumn{8}{|c|}{ Predicted (No. of trips) } \\
\hline \multirow{7}{*}{$\begin{array}{c}\text { Observed } \\
\text { (No. of trips) }\end{array}$} & & Walk & Auto-rickshaw & Bus & Two-wheeler & Car & Total \\
\hline & Walk & 17 & 2 & 0 & 0 & 0 & 19 \\
\hline & Auto-rickshaw & 0 & 24 & 3 & 0 & 0 & 27 \\
\hline & Bus & 0 & 0 & 19 & 0 & 0 & 19 \\
\hline & Two-wheeler & 0 & 2 & 1 & 46 & 0 & 49 \\
\hline & Car/School van & 0 & 0 & 0 & 3 & 6 & 9 \\
\hline & Total & 17 & 28 & 23 & 49 & 6 & 123 \\
\hline
\end{tabular}

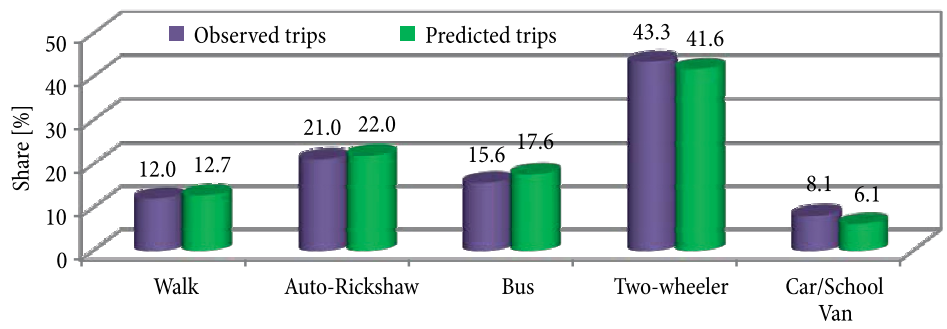

Fig. 7. Comparison of field observed and model predicted modal split

urban scenario. The developed model can be used to assess the effectiveness of the transport policies before implementation.

\subsection{Mode Choices: Income Impact}

As the vehicle ownership is a function of household income, composition etc. the set of available alternatives for travel mode varies among different socio-economic classes of the society. Table 5 shows the observed and predicted mode usage pattern for different income groups considered in this study. Predicted values are very close to the survey data. Walking share reduces drastically with higher income group. Share of twowheelers are almost same for MIG and HIG. The share of school bus is high for HIG, High comfort and safety in school buses may be the reason behind it whereas share of auto-rickshaw is high for MIG.

\section{Sensitivity Analysis}

\subsection{Land-use planning: Reduction in Trip Length}

Land-use planning if done in such a way that the academic institutions are properly placed with reference to the residential neighbourhoods particularly for pre-primary

Table 5. Income wise comparison of observed and predicted modal split

\begin{tabular}{|c|c|c|c|c|c|c|}
\hline \multirow{3}{*}{ Mode } & \multicolumn{6}{|c|}{ Share of trips [\%] } \\
\hline & \multicolumn{2}{|c|}{ LIG } & \multicolumn{2}{|c|}{ MIG } & \multicolumn{2}{|c|}{ HIG } \\
\hline & Observed & Predicted & Observed & Predicted & Observed & Predicted \\
\hline Walk & 20.4 & 34.7 & 15.9 & 15.3 & 5.3 & 3.5 \\
\hline Auto-rickshaw & 36.7 & 24.5 & 21.2 & 26.0 & 16.4 & 17.0 \\
\hline Bus & 6.1 & 8.2 & 12.2 & 14.3 & 22.2 & 24.0 \\
\hline Two-wheeler & 30.7 & 30.6 & 46.0 & 41.8 & 43.8 & 44.4 \\
\hline Car/School van & 6.1 & 2.0 & 4.7 & 2.6 & 12.3 & 11.1 \\
\hline
\end{tabular}


and primary schooling level, it encourage the non-motor vehicle travel because of reduced trip lengths. Similarly, it is important to note the locations for secondary and higher secondary level of schooling. Strengthening the infrastructure for non-motorized transport is possible at Town Planning Scheme and Neighbourhood levels. In effect, Share by walking and cycling is bound to rise and reduction in auto trips resulting thereby in fuel savings, pollution levels and traffic hazards.

Considering $50 \%$ reduction in trip length of the data used as a typical hypothetical case, one can realize the impact on the modal share. The share of auto-rickshaw, two-wheeler and car is likely to decrease specially for LIG and MIG whereas the share of walking mode is observed to increase considerably. People belonging to higher income group are less likely to be affected by the reduced trip lengths. It may be because of their status issues and higher comfort and safety desired by them. Results are comparable to reflect on the impact as shown in Table 6.

Table 6. Income wise impact of land-use planning on modal split

\begin{tabular}{lcc|cc|cc}
\hline \multirow{2}{*}{ Mode } & \multicolumn{6}{c}{ Share of trips [\%] } \\
\cline { 2 - 8 } & \multicolumn{2}{c}{ LIG } & \multicolumn{2}{c}{ MIG } & \multicolumn{2}{c}{ HIG } \\
\cline { 2 - 8 } & before & after & before & after & before & after \\
\hline Walk & 20.4 & 51.0 & 15.9 & 24.9 & 5.3 & 3.5 \\
\hline Auto-rickshaw & 36.7 & 22.4 & 21.2 & 20.1 & 16.4 & 15.8 \\
\hline Bus & 6.1 & 8.2 & 12.2 & 14.3 & 22.2 & 23.4 \\
\hline Two-wheeler & 30.7 & 18.4 & 46.0 & 38.6 & 43.8 & 46.2 \\
\hline Car/School van & 6.1 & 0 & 4.7 & 2.1 & 12.3 & 11.1 \\
\hline
\end{tabular}

\section{Summary and Conclusions}

Modal choice is as such third stage process in urban transportation planning package. Mode choice is associated with socio-economic, modal, trip and network characteristics. Logit modelling in this regard is a popular conventional approach based on crisp inputs. In reality, it depends on the judgement by the traveller perception and in linguistic expressions, wherein the boundaries are not fixed and uncertainty prevails. Hence, fuzzy rule-based mode choice model is considered as the better option to address the shortcomings in crisp based modelling. The developed model (FRBMCM-E) finds applications in assessment of effectiveness of policies such as strengthening the infrastructure for non-motorized modes as well as improvement in operational and functional features of existing travel modes. Effective management of traffic flow on urban roads and transportation system as a whole can be facilitated by these models with due considerations to various income classes in fast growing Indian cities.

\section{Acknowledgements}

Authors thank all the respondents of the Household Interview Surveys conducted in Surat city for giving their precious feedbacks without which it was not possible to conduct this study.
In addition, authors are thankful to Dr G. J. Joshi for facilitating the research study being the Section Head at P. G. Section in Transportation Engineering and Planning, S. V. National Institute of Technology, Surat, India.

\section{References}

Bataineh, K. M.; Naji, M.; Saqer, M. 2011. A comparison study between various fuzzy clustering algorithms, Jordan Journal of Mechanical and Industrial Engineering 5(4): 335-343.

Dell'Orco, M.; Ottomanelli, M. 2012. Simulation of users decision in transport mode choice using neuro-fuzzy approach, Lecture Notes in Computer Science 7334: 44-53. http://dx.doi.org/10.1007/978-3-642-31075-1_4

Errampalli, M.; Okushima, M.; Akiyama, T. 2011. Fuzzy Logic based Microscopic Traffic Simulation Model: Simulation of Vehicles and Commuters on Urban Road Network to Evaluate Transport Policies. VDM Verlag Dr. Müller. 288 p.

Holland, R. 2000. Fuzzy logic model of mode choice, in Proceedings of Seminar of the European Transport Conference 2000, 11-13 September 2000, Cambridge, UK, 41-53. Available from Internet: http://abstracts.aetransport.org/ paper/index/id/1147/confid/6

Jassbi, J.; Alavi, S. H.; Serra, P. J. A.; Ribeiro, R. A. 2007. Transformation of a Mamdani FIS to first order Sugeno FIS, in FUZZ-IEEE 2007: IEEE International Fuzzy Systems Conference 2007, 23-26 July 2007, London, UK, 1-6. http://dx.doi.org/10.1109/FUZZY.2007.4295331

Kalić, M.; Teodorović, D. 2003. Trip distribution modelling using fuzzy logic and a genetic algorithm, Transportation Planning and Technology 26(3): 213-238. http://dx.doi.org/10.1080/0308106032000154575

Kalić, M.; Teodorović, D. 1996. Solving the trip distribution problem by fuzzy rules generated by learning from examples, in Proceedings of the XXIII Yugoslav Symposium on Operations Research, 1-5 October 1996, Zlatibor, Yugoslavia, 777-780. (in Serbian).

Kompil, M.; Celik, H. M. 2013. Modelling trip distribution with fuzzy and genetic fuzzy systems, Transportation Planning and Technology 36(2): 170-200.

http://dx.doi.org/10.1080/03081060.2013.770946

Kumar, M.; Sarkar, P.; Errampalli, M. 2013. Development of fuzzy logic based mode choice model considering various public transport policy options, International Journal for Traffic and Transport Engineering 3(4): 408-425. http://dx.doi.org/10.7708/ijtte.2013.3(4).05

Mamdani, E. H.; Assilian, S. 1975. An experiment in linguistic synthesis with a fuzzy logic controller, International Journal of Man-Machine Studies 7(1): 1-13. http://dx.doi.org/10.1016/S0020-7373(75)80002-2

Mendel, J. M. 2001. Uncertain Rule-Based Fuzzy Logic Systems: Introduction and New Directions. Prentice Hall. 576 p.

Mizutani, K.; Akiyama, T. 2000. A logit model for modal choice with a fuzzy logic utility function, in Traffic and Transportation Studies (2000): Proceedings of the Second International Conference, 31 July - 2 August 2000, Beijing, China, 311-318. http://dx.doi.org/10.1061/40503(277)49

Murat, Y. S.; Uludag, N. 2008. Route choice modelling in urban transportation networks using fuzzy logic and logistic regression methods, Journal of Scientific and Industrial Research 67(1):19-27.

Pulugurta, S.; Arun, A.; Errampalli, M. 2013. Use of artificial intelligence for mode choice analysis and comparison with 
traditional multinomial logit model, Procedia - Social and Behavioral Sciences 104: 583-592.

http://dx.doi.org/10.1016/j.sbspro.2013.11.152

Pulugurta, S.; Errampalli, M.; Kayitha, R. 2014. Fuzzy logicbased travel demand model to simulate public transport policies, Journal of Urban Planning and Development. http://dx.doi.org/10.1061/(ASCE)UP.1943-5444.0000261

Teodorović, D. 1999. Fuzzy logic systems for transportation engineering: the state of the art, Transportation Research Part A: Policy and Practice 33(5): 337-364.

http://dx.doi.org/10.1016/S0965-8564(98)00024-X

Vythoulkas, P. C.; Koutsopoulos, H. N. 2003. Modeling discrete choice behavior using concepts from fuzzy set theory, approximate reasoning and neural networks, Transportation Research Part C: Emerging Technologies 11(1): 51-73. http://dx.doi.org/10.1016/S0968-090X(02)00021-9

Wang, L.-X.; Mendel, J. M. 1992. Generating fuzzy rules by learning from examples, IEEE Transactions on Systems, Man and Cybernetics 22(6): 1414-1427. http://dx.doi.org/10.1109/21.199466

Zadeh, L. A. 1965. Fuzzy sets, Information and Control 8(3): 338353. http://dx.doi.org/10.1016/S0019-9958(65)90241-X 